\title{
Nucleon mass and pion-nucleon sigma term from a chiral analysis of lattice QCD world data
}

\author{
L. Alvarez-Ruso ${ }^{1}$, T. Ledwig ${ }^{2, a}$, J. Martin Camalich ${ }^{3}$ and M.J. Vicente Vacas ${ }^{2}$ \\ ${ }^{1}$ Instituto de Física Corpuscular (IFIC), Centro Mixto Universidad de Valencia-CSIC, Valencia, Spain \\ ${ }^{2}$ Departamento de Física Teórica and IFIC, Centro Mixto Universidad de Valencia-CSIC, Valencia, \\ Spain \\ ${ }^{3}$ Department of Physics and Astronomy, University of Sussex, Brighton, UK
}

\begin{abstract}
The chiral behavior of the nucleon mass is studied within the covariant $S U(2)$ baryon chiral perturbation theory up to order $p^{4}$. Lattice QCD data for the ensembles of 2 and $2+1$ flavors are separately fitted, paying special attention to explicit $\Delta(1232)$ degrees of freedom, finite volume corrections and finite spacing effects. In the case of the 2 flavor ensemble, we fit simultaneously nucleon mass data together with new and updated data for the $\sigma_{\pi N}$ term both in their dimensionless forms and determine a Sommer-scale of $r_{0}=0.493(23) \mathrm{fm}$. We obtain low-energy constants of natural size that are compatible with the rather linear pion-mass dependence observed in lattice QCD and report a preliminary updated value of $\sigma_{\pi N}=43(5)(4) \mathrm{MeV}$ for the 2 flavor case and $\sigma_{\pi N}=52(3)(8) \mathrm{MeV}$ for $2+1$ flavor case.
\end{abstract}

\section{Introduction}

Only lattice QCD (1QCD) simulations and baryon chiral perturbation theory (B $\chi \mathrm{PT})$ can presently address low-energy hadron observables in a systematically improvable manner. In both frameworks they can be studied for quark masses $\left(m_{u}=m_{d}=\bar{m}\right)$ not restricted to their physical values. This allows for combined analyses where $1 \mathrm{QCD}$ data points for the unphysical region can be fitted by $\mathrm{B} \chi \mathrm{PT}$ results, setting the scale in the former [1] and fixing low-energy constants (LECs) of the latter; LECs that afterwards can be used for $\mathrm{B} \chi \mathrm{PT}$ predictions at the physical point.

In [2] we performed such a matching of the quark-mass (or pion-mass $M_{\pi}^{2} \sim \bar{m}$ ) dependence of the nucleon mass, $M_{N}(\bar{m})$, of the $S U(2) p^{4}$ covariant B $\chi \mathrm{PT}$ to $1 \mathrm{QCD}$ world data for two degenerated light quarks $\left(N_{f}=2\right)[1,3-6]$ or two degenerated light and one heavy quark $\left(N_{f}=2+1\right)$ [7-13].

Additionally, this dependence is related to the $\sigma_{\pi N}$ term via the Hellmann-Feynman theorem:

$$
\bar{m} \frac{\partial}{\partial \bar{m}} M_{N}(\bar{m})=\sigma_{\pi N}=\bar{m}\langle N|\bar{u} u+\bar{d} d| N\rangle
$$

\footnotetext{
${ }^{a}$ e-mail: ledwig@ific.uv.es
}

This is an Open Access article distributed under the terms of the Creative Commons Attribution License 4.0, which permits unrestricted use, distribution, and reproduction in any medium, provided the original work is properly cited. 
which relates $M_{N}(\bar{m})$ to the nucleon scalar form factor at zero momentum transfer squared. Therefore indirectly also to $\pi N$-scattering experiments with $\sigma_{\pi N}=59$ (7) $\mathrm{MeV}$ [14] as the latest result. Together with the LECs extracted by the B $\chi \mathrm{PT} / \mathrm{lQCD}$ fitting, Eq. (1) enables $\mathrm{B} \chi \mathrm{PT}$ predictions for the $\sigma_{\pi N}$ term at the physical point. Scalar matrix elements as in Eq. (1) are important for current dark matter searches.

\section{Nucleon mass and covariant baryon chiral perturbation theory}

We use the $S U(2) p^{4}$ covariant $\mathrm{B} \chi \mathrm{PT}$ with explicit $\Delta(1232)$ degrees of freedom in the EOMS renormalization [15] to parametrize the chiral structure of the nucleon mass. The result is written as:

$$
M_{N}^{(4)}\left(M_{\pi}^{2}\right)=M_{0}-c_{1} 4 M_{\pi}^{2}+\frac{1}{2} \bar{\alpha} M_{\pi}^{4}+\frac{c_{1}}{8 \pi^{2} f_{\pi}^{2}} M_{\pi}^{4} \ln \frac{M_{\pi}^{2}}{M_{0}^{2}}+\Sigma_{\text {loops }}^{(3)+(4)}\left(c_{1}, M_{\pi}^{2}\right)+\mathcal{O}\left(p^{5}\right) .
$$

with $M_{\pi}^{2} \sim \bar{m}$ and $f_{\pi}$ as the pion mass and pion decay constant. The $\Delta(1232)$ contributions enter through the pion loops which are given in [2] in detail. After fitting the three LECs $M_{0}, c_{1}$ and $\bar{\alpha}$ to the 1QCD data, we extract via Eq. (1) a $\sigma_{\pi N}$ value at the physical point. For all fits we include finite volume correction (box size $L$ ) and additionally study finite spacing $(a)$ effects which we parametrize through action-specific constants $c_{a} a^{2}$ in the $\chi^{2}$ function.

We fit the two data sets of the $N_{f}=2$ and $N_{f}=2+1$ ensembles separately. In the case of $N_{f}=2$, we use the dimensionless form $\left(r_{0} M_{\pi}, r_{0} M_{N}\right)$, with $r_{0}$ being the Sommer-scale which we obtain simultaneously during the fit. For the $N_{f}=2+1$ case, we use the scales as given by each collaboration and consider scale-introduced data correlation by defining a correlation matrix for the $\chi^{2}$. In our $S U(2) \mathrm{B} \chi \mathrm{PT}$ approach, we treat the strange quark effects as being integrated out and absorbed into the LECs. For both ensembles, we only use data points fulfilling $M_{\pi} L>3.8$ and $r_{0} M_{\pi}<1.11$ or $M_{\pi}<415 \mathrm{MeV}$, respectively, to ensure controlled finite volume effects and an acceptable chiral convergence. Additionally, the QCDSF collaboration is releasing new direct $\sigma_{\pi N}$ data points $[1,6]$ which we include in our $N_{f}=2$ fits.

\section{Results and conclusions}

We summarize here our results of Ref. [2]. In the case of the $N_{f}=2$ ensemble fits, we perform a preliminary actualization by using here the updated $\sigma_{\pi N}$ data points of [6], Fig. 1, without the preliminary data point at $M_{\pi} \approx 150 \mathrm{MeV}$.

Including or excluding the direct calculations of the $\sigma_{\pi N}$ influences the $N_{f}=2$ fits without explicit $\Delta(1232)$ contributions noticeably. Uncertainties for the nucleon mass description are reduced by the former, which lies in the uncertainty range of the latter. However, the slope is changed. This is also seen in the obtained $\sigma_{\pi N}$ value at the physical point which changes from 62(13) to 45(3) MeV. The situation is different when we also include the explicit $\Delta(1232)$ contributions in our fits. For this case, the inclusion of the $\sigma_{\pi N}$ data points does not change the pion-mass dependence much and changes the $\sigma_{\pi N}$ value at the physical point only from $38(10)$ to $42(2) \mathrm{MeV}$. This might be interpreted as an indication that the theory with explicit $\Delta(1232)$ is more realistic. Following the same arguments for the error definition as in [2], we give here a preliminary updated $N_{f}=2$ value of the $\sigma_{\pi N}$ value at the physical point of:

$$
\sigma_{\pi N}^{N_{f}=2}=43(5)(4) \mathrm{MeV} .
$$

The Fig. 2 shows the pion-mass dependence of the nucleon mass and the $\sigma_{\pi N}$ term obtained from our B $\chi$ PT fits to the $N_{f}=2+1$ data. In general, all fit strategies, i.e. in-/ excluding $\Delta(1232)$ degrees of freedom or finite spacing parametrization, give a consistent description for the nucleon mass. However, the small slope variations turn into noticeable different predictions for the $\sigma_{\pi N}$ term. For successively including $\Delta(1232)$ contributions and finite spacing parametrization, the $\sigma_{\pi N}$ value at the physical point changes by up to $14 \mathrm{MeV}$ from $\sigma_{\pi N}=58(3)$ to $49(2)$ down to $44(3) \mathrm{MeV}$. To give a final value, we take 

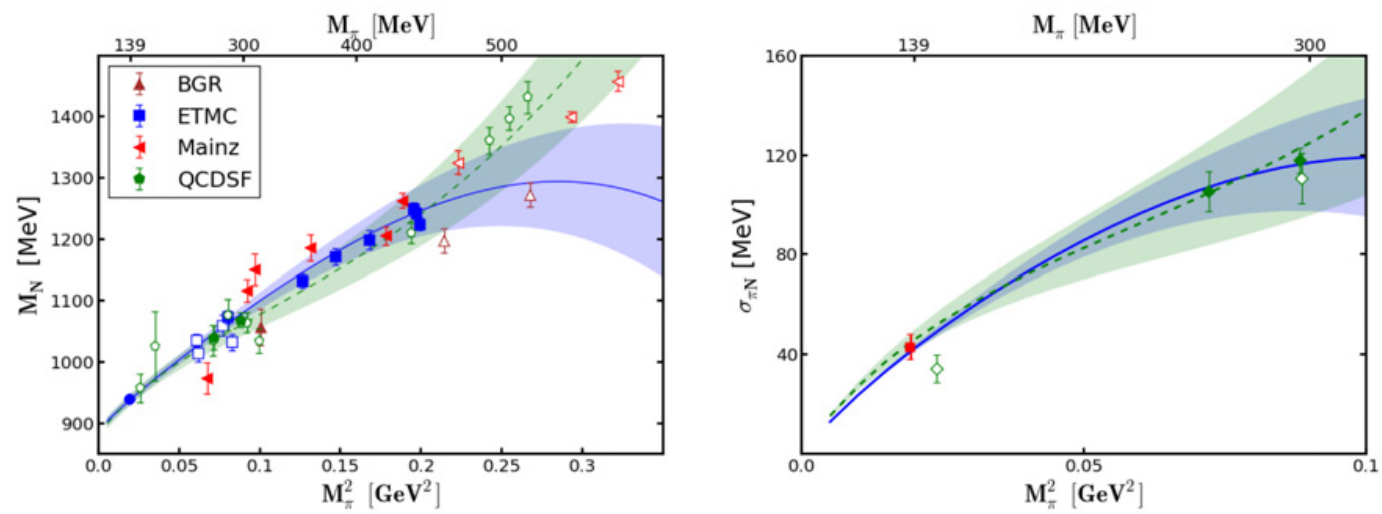

Figure 1. Pion-mass dependence of the nucleon mass and the $\sigma_{\pi N}$ term from our $N_{f}=2$ fits. The $1 \mathrm{QCD}$ data points are scaled by the $r_{0}$-value as obtained in the $\Delta(1232)$ fits (blue-solid lines) and results without $\Delta(1232)$ (green-dashed lines) are shown for comparison. Data points denoted by open markers are not included in the fit. The $\sigma_{\pi N}$ data points are from [1], open marker at $M_{\pi} \approx 290 \mathrm{MeV}$, and the updates from [6] with the preliminary point at $M_{\pi} \approx 150 \mathrm{MeV}$. The blue circle and the red square are the experimental nucleon mass and our $\sigma_{\pi N}$ estimate at the physical point.
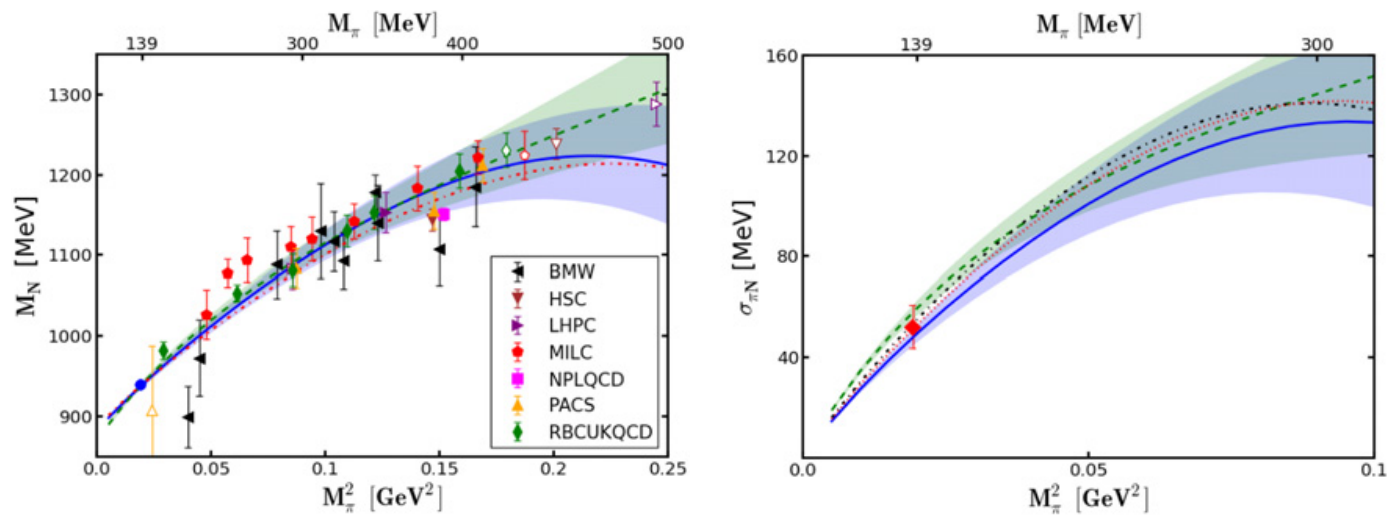

Figure 2. Pion-mass dependence of the nucleon mass and the $\sigma_{\pi N}$ term from our $N_{f}=2+1$ fits. Blue-solid lines correspond to including explicit $\Delta(1232)$ degrees of freedom, green-dashed ones to excluding them. Red and black lines correspond to fit choices explained in [2]. Data points denoted by open markers are not included in the fit. The blue circle and the red square are the experimental nucleon mass and our $\sigma_{\pi N}$ estimate at the physical point.

a conservative standpoint and take a weighted average, resulting in:

$$
\sigma_{\pi N}^{N_{f}=2+1}=52(3)(8) \mathrm{MeV},
$$

where the second (systematical) uncertainty spans all the above central values.

Both values are compatible within the uncertainties. However, even though with the new $N_{f}=2$ data reducing the spread slightly, a difference of $9 \mathrm{MeV}$ of the central values remains. With the present data situation, several explanations are possible. First, the $N_{f}=2$ data does not constrain the low pionmass region as much as the $N_{f}=2+1$ data does. This results in an up to $\sim 10 \%$ different $c_{1}$ value which determines solely the $\sigma_{\pi N}$ at leading order. Secondly, direct lQCD calculations of the $\sigma_{\pi N}$ influence the $N_{f}=2$ fits noticeably. Especially, the $\Delta(1232)$ and $\Delta(1232)$-less results are brought together. For the $N_{f}=2+1$ case, such low- $M_{\pi} \sigma_{\pi N}$ data points are not available. Thirdly, the strange quark is treated 
differently in the two fit versions and one could expect that part of the difference might be due to this fact.

In summary, we used the $S U(2) p^{4}$ covariant $\mathrm{B} \chi \mathrm{PT}$ to fitt $1 \mathrm{QCD}$ data of the $N_{f}=2$ and $N_{f}=2+1$ ensembles. We take special care of systematic uncertainties, such as explicit $\Delta(1232)$ degrees of freedom, finite volume and finite spacing effects, as well as LQCD scale setting or data correlation. Even though the present data set is extensive, we find systematic uncertainties stemming mostly from the distribution of the data points. It will be interesting to see how new $N_{f}=2$ data in the low- $M_{\pi}$ region or especially $N_{f}=2+1$ data points for the $\sigma_{\pi N}$ with $M_{\pi}<300 \mathrm{MeV}$ will influence the fits.

The work has been supported by the Spanish Ministerio de Economía y Competitividad and European FEDER funds under Contracts FIS2011-28853-C02-01 and FIS2011-28853-C02-02, Generalitat Valenciana under contract PROMETEO/2009/0090 and the EU Hadron-Physics3 project, Grant No. 283286. JMC also acknowledges support from the Science Technology and Facilities Council (STFC) under grant ST/J000477/1, the grants FPA2010-17806 and Fundación Séneca 11871/PI/09.

\section{References}

[1] G.S. Bali, P.C. Bruns, S. Collins, M. Deka, B. Glasle, M. Gockeler, L. Greil, T.R. Hemmert, R. Horsley, J. Najjar, Nucl. Phys. B 866, 1 (2013)

[2] L. Alvarez-Ruso, T. Ledwig, J. Martin-Camalich, M.J. Vicente-Vacas, Phys. Rev. D 88, 054507 (2013)

[3] G.P. Engel et al. (BGR Collaboration), Phys. Rev. D 82, 034505 (2010)

[4] C. Alexandrou et al. (ETM Collaboration), Phys. Rev. D 83, 045010 (2011)

[5] S. Capitani, M. Della Morte, G. von Hippel, B. Jager, A. Juttner, B. Knippschild, H.B. Meyer, H. Wittig Phys. Rev. D 86, 074502 (2012)

[6] G.S. Bali, S. Collins, B. Gláßle, M. Göckeler, J. Najjar, R. Rödl, A. Schäfer, R. Schiel, W. Söldner, A. Sternbeck, PoS Lattice2013, 291 (2013)

[7] S. Durr et al. (BMW Collaboration), Phys. Rev. D 85, 014509 (2012)

[8] H.-W. Lin et al. (HS Collaboration), Phys. Rev. D 79, 034502 (2009)

[9] J.D. Bratt et al. (LHPC Collaboration), Phys. Rev. D 82, 094502 (2010)

[10] C. Aubin, C. Bernard, C. DeTar, J. Osborn, Steven Gottlieb, E.B. Gregory, D. Toussaint, U.M. Heller, J.E. Hetrick, R. Sugar, Phys. Rev. D 70, 094505 (2004); D. Toussaint et al. (MILC Collaboration), private communication

[11] S.R. Beane et al. (NPLQCD Collaboration), Phys. Rev. D 84, 014507 (2011)

[12] S. Aoki et al. (PACS Collaboration), Phys. Rev. D 79, 034503 (2009)

[13] C. Jung et al. (RBC and UKQCD Collaborations), PoS LATTICE2012, 164 (2012)

[14] J.M. Alarcon, J. Martin Camalich, J.A. Oller, Phys. Rev. D 85, 051503 (2012)

[15] J. Gegelia, G. Japaridze, Phys. Rev. D 60, 114038 (1999); T. Fuchs, J. Gegelia, G. Japaridze, S. Scherer, Phys. Rev. D 68, 056005 (2003) 